\title{
Author Correction: Loss of MTX2 causes mandibuloacral dysplasia and links mitochondrial dysfunction to altered nuclear morphology
}

Sahar Elouej, Karim Harhouri (1D, Morgane Le Mao, Genevieve Baujat, Sheela Nampoothiri, Hülya Kayserili (D), Nihal Al Menabawy (D), Laila Selim, Arianne Llamos Paneque, Christian Kubisch, Davor Lessel(D),

Robert Rubinsztajn, Chayki Charar, Catherine Bartoli, Coraline Airault, Jean-François Deleuze (D), Agnes Rötig (D), Peter Bauer, Catarina Pereira, Abigail Loh, Nathalie Escande-Beillard (D), Antoine Muchir (D), Lisa Martino, Yosef Gruenbaum, Song-Hua Lee $\mathbb{D}$, Philippe Manivet $\mathbb{D}$, Guy Lenaers, Bruno Reversade $\mathbb{D}$, Nicolas Lévy \& Annachiara De Sandre-Giovannoli (i)

Correction to: Nature Communications https://doi.org/10.1038/s41467-020-18146-9, published online 11 September 2020

The original version of this Article contained errors in the author affiliations.

The affiliations of Nathalie Escande-Beillard and Bruno Reversade with 'Medical Genetics Department, Koç University, School of Medicine (KUSoM), Istanbul, Turkey' were inadvertently omitted.

This has now been corrected in both the PDF and HTML versions of the Article.

Published online: 19 October 2020

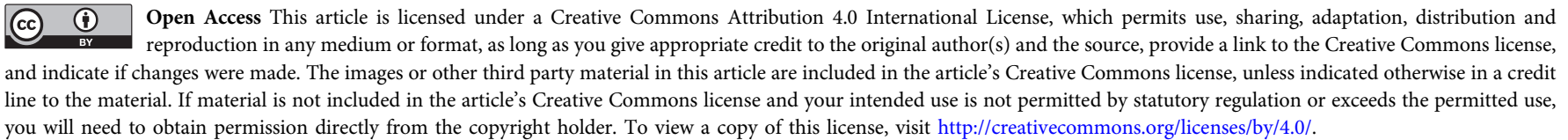

(C) The Author(s) 2020 